\title{
A parsimonious explanation for intersecting perinatal mortality curves: understanding the effect of plurality and of parity
} KS Joseph*1, Shiliang Liu² ${ }^{2}$ Kitaw Demissie ${ }^{3}$, Shi Wu Wen ${ }^{2}$, Robert W Platt ${ }^{4}$, Cande V Ananth ${ }^{5}$, Susie Dzakpasu ${ }^{2}$, Reg Sauve ${ }^{6}$, Alexander C Allen ${ }^{1}$, Michael S Kramer ${ }^{4}$ and the Fetal and Infant Health Study Group of the Canadian Perinatal Surveillance System ${ }^{7}$

\begin{abstract}
Address: ${ }^{1}$ Perinatal Epidemiology Research Unit, Departments of Obstetrics \& Gynecology and of Pediatrics, Dalhousie University, Halifax, Nova Scotia, Canada, ${ }^{2}$ Health Surveillance and Epidemiology Division, Centre for Healthy Human Development, Health Canada, Ottawa, Ontario, Canada, ${ }^{3}$ Department of Environmental and Community Medicine, Robert Wood Johnson Medical School, University of Medicine and Dentistry of New Jersey, Piscataway, NJ, USA, ${ }^{4}$ Departments of Pediatrics and of Epidemiology and Biostatistics, McGill University, Montreal, Quebec, Canada, 5 Section of Epidemiology and Biostatistics, Department of Obstetrics, Gynecolgy and Reproductive Sciences, Robert Wood Johnson Medical School, University of Medicine and Dentistry of New Jersey, Piscataway, NJ, USA, ${ }^{6}$ Departments of Pediatrics and of Community Health, University of Calgary, Calgary, Alberta, Canada and ${ }^{7}$ Canadian Perinatal Surveillance System, Health Surveillance and Epidemiology Division, Centre for Healthy Human Development, Health Canada, Ottawa, Ontario, Canada
\end{abstract}

Email: KS Joseph* - kjoseph@is.dal.ca; Shiliang Liu - shiliang_liu@hc-sc.gc.ca; Kitaw Demissie - demisski@umdnj.edu; ShiWuWen - swwen@ohri.ca; RobertW Platt - robert.platt@mcgill.ca; Cande V Ananth - ananthcv@epi.umdnj.edu; Susie Dzakpasu - susie_dzakpasu@hc-sc.gc.ca; Reg Sauve - rsauve@acs.ucalgary.ca; Alexander C Allen - ac.allen@np.iwkgrace.ns.ca; Michael S Kramer - michael.kramer@mcgill.ca; the Fetal and Infant Health Study Group of the Canadian Perinatal Surveillance System -

* Corresponding author

Published: 2 June 2003

BMC Pregnancy and Childbirth 2003, 3:3
Received: 18 December 2002

Accepted: 2 June 2003

This article is available from: http://www.biomedcentral.com/I47/-2393/3/3

(c) 2003 Joseph et al; licensee BioMed Central Ltd. This is an Open Access article: verbatim copying and redistribution of this article are permitted in all media for any purpose, provided this notice is preserved along with the article's original URL.

\begin{abstract}
Background: Birth weight- and gestational age-specific perinatal mortality curves intersect when compared across categories of maternal smoking, plurality, race and other factors. No simple explanation exists for this paradoxical observation.
\end{abstract}

Methods: We used data on all live births, stillbirths and infant deaths in Canada (199|-1997) to compare perinatal mortality rates among singleton and twin births, and among singleton births to nulliparous and parous women. Birth weight- and gestational age-specific perinatal mortality rates were first calculated by dividing the number of perinatal deaths at any given birth weight or gestational age by the number of total births at that birth weight or gestational age (conventional calculation). Gestational age-specific perinatal mortality rates were also calculated using the number of fetuses at risk of perinatal death at any given gestational age.

Results: Conventional perinatal mortality rates among twin births were lower than those among singletons at lower birth weights and earlier gestation ages, while the reverse was true at higher birth weights and later gestational ages. When perinatal mortality rates were based on fetuses at risk, however, twin births had consistently higher mortality rates than singletons at all gestational ages. A similar pattern emerged in contrasts of gestational age-specific perinatal mortality among singleton births to nulliparous and parous women. Increases in gestational age-specific rates of growth-restriction with advancing gestational age presaged rising rates of gestational age-specific perinatal mortality in both contrasts.

Conclusions: The proper conceptualization of perinatal risk eliminates the mortality crossover paradox and provides new insights into perinatal health issues. 


\section{Background}

Over 30 years ago, Yerushalmy identified a paradoxical relationship between maternal smoking and birth weightspecific neonatal mortality [1]. Neonatal deaths rates among infants of smokers were lower than among infants of non-smokers at birth weights of $3000 \mathrm{~g}$ or less; the reverse was true at higher birth weights. In the last $3 \mathrm{dec}-$ ades, this observation has been confirmed as a general phenomenon $[2,3]$ which emerges when birth weight- or gestational age-specific perinatal mortality curves are compared across plurality, race, parity, infant sex and country [2-10].

Intersecting perinatal mortality curves present an inferential challenge. The argument that infants of women who smoke during pregnancy (or twin infants) are healthier than the infants of non-smokers (or singleton infants) at some birth weights but not others lacks biological plausibility and coherence. Sophisticated statistical approaches [3-11] have been proposed to address this problem and include explanations based on 'relative birth weight' [4] or 'relative gestational age' [10]. For instance, Wilcox and Russell [4] showed that examining perinatal mortality rates across categories of relative birth weight (i.e., expressing birth weight in terms of the population mean and standard deviation) eliminates the crossover phenomenon.

The relative birth weight formulation has been criticized because it fails to distinguish between birth weight differences due to maturity (i.e., higher gestational age) versus fetal growth (i.e., birth weight for gestational age) $[12,13]$. However, the most serious criticism of the relative birth weight and relative gestational age techniques is that they do not represent a parsimonious solution. Although the use of relative birth weight [4] and relative gestational age [3] resolves the perinatal mortality crossover, these approaches carry onerous implications for epidemiologic and statistical modeling in medical research where relative values of determinants (such as adult age or weight) are rarely used. In this paper, we propose a parsimonious solution to the paradox presented by intersecting perinatal mortality curves.

\section{Methods}

We used data on all live births and stillbirths in Canada from Statistics Canada's live births and stillbirths databases for the years 1991 to 1997 and data for all infant deaths from the mortality database for the years 1991 to 1998. Information in these databases is obtained from live birth, stillbirth and death registrations supplied by Canadian provincial and territorial vital statistics registries [14]. Information on gestational age in the live birth registration is obtained from the physician responsible for the delivery or the mother, while the stillbirth registration is completed by a physician. Although the method of gestational age ascertainment is not stated in birth registration documents, previous research suggests that gestational age information in the database is reliable and increasingly based on early ultrasound dating, especially in recent years $[15,16]$. The live birth and mortality databases have been previously linked in order to obtain perinatal information on infant deaths. The linked file has been subjected to data quality checks in order to eliminate duplicate records and the completeness and validity of the linked data has been verified [17]. Births to mothers residing in Ontario were excluded because of documented problems with data quality [18] and significant numbers of unlinked infant deaths.

We first compared the gestational age and birth weight distributions of singleton and twin live births. We then contrasted gestational age- and birth weight-specific stillbirth, early neonatal death ( 0 to 6 days) and perinatal mortality (stillbirths plus early neonatal deaths) rates among singleton and twin births. For these analyses, gestational age- and birth weight-specific perinatal mortality rates were calculated (as per convention) by dividing the number of perinatal deaths at any given gestational age or birth weight by the number of total births (stillbirths and live births) at that gestational age or birth weight.

Subsequent analyses were carried out with the gestational age-specific stillbirth rate calculated as the number of stillbirths at any gestation divided by the number of fetuses at risk of stillbirth at that gestation. For instance, the denominator for calculating the stillbirth rate at 32 weeks gestation included all births at 32 weeks and over. This implies that fetuses at 32 weeks who go on to deliver at 33 weeks or over are potential candidates for stillbirth at 32 weeks. We calculated gestational age-specific early neonatal death rates and gestational age-specific perinatal mortality rates using the same denominator we used for calculating gestational age-specific stillbirth rates. This implies that a fetus at 32 weeks gestation is at risk for birth and for early neonatal death at that gestation. Stillbirths were viewed as competing risks (non-independent) in analyses of early neonatal death [19].

In order to explain gestational age-specific patterns of mortality, we also calculated gestational age-specific patterns of birth ('birth rates') and fetal growth-restriction using fetuses at risk as the denominator. Growth restricted fetuses were identified as those live births with a birth weight for gestational age less than the $10^{\text {th }}$ percentile of the recently published Canadian fetal growth standard [20]. We repeated all analyses using parity (singleton births to nulliparous vs parous women) as the determinant across which perinatal mortality curves intersect. 

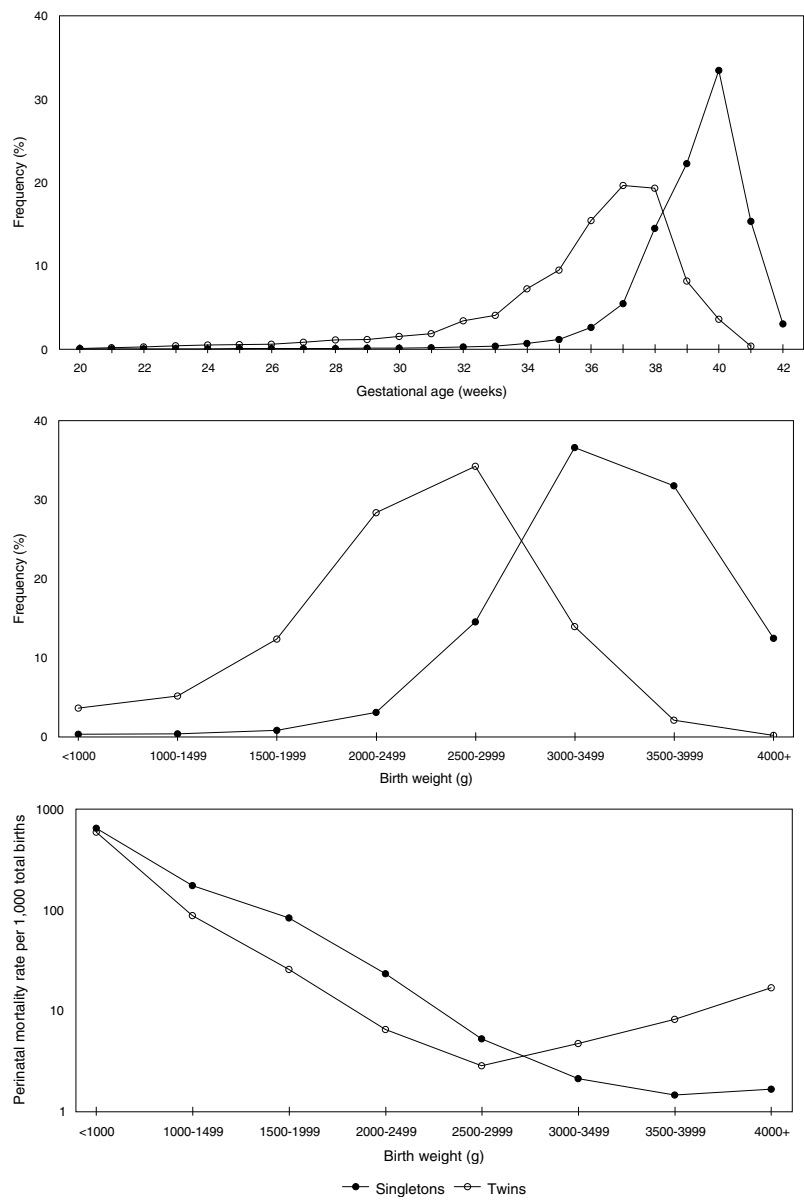

Figure I

Gestational age, birth weight and birth weight-specific perinatal mortality among singletons and twins. Legend text: Gestational age (upper panel) and birth weight (middle panel) distributions and birth weight-specific perinatal mortality rates (lower panel) among singleton and twin births, Canada (excluding Ontario), 1991-97.

\section{Results}

The gestational age and birth weight distributions of twin live births were 'shifted to the left' as compared with corresponding distributions of singleton live births (Figure 1 ). The stillbirth rate among singleton births was 5.4 per 1,000 total births, the early neonatal death rate was 2.8 per 1,000 live births and the perinatal mortality rate was 8.2 per 1,000 total births. In contrast, twins experienced substantially higher rates of stillbirth (19.7 per 1,000 total births), early neonatal death (21.5 per 1,000 live births) and perinatal mortality (40.8 per 1,000 total births). Among singletons, 71 percent of perinatal deaths occurred at preterm gestation ( $<37$ weeks), while this proportion was 92 percent among twins.
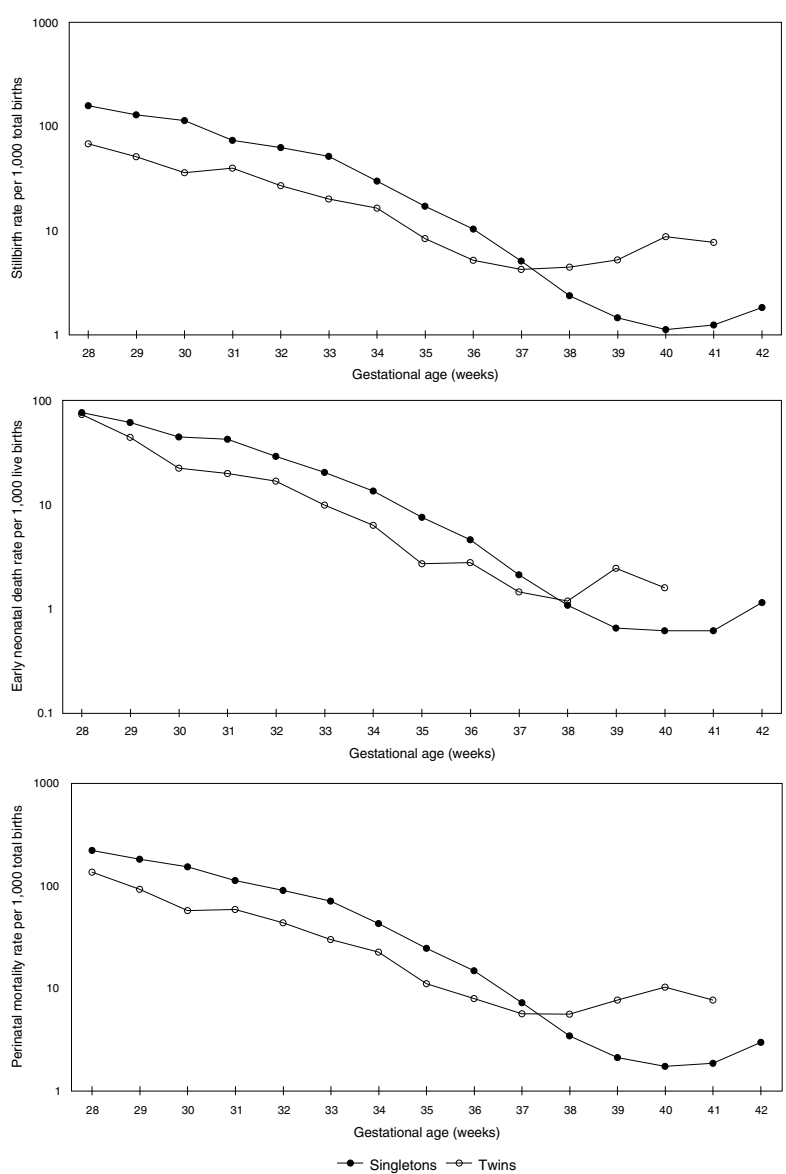

Figure 2

Stillbirth, early neonatal death and perinatal mortality rates among singleton and twin births (per convention). Legend text: Gestational age-specific stillbirth (upper panel), early neonatal death (middle panel) and perinatal mortality (lower panel) rates among singleton and twin births calculated as per convention (i.e., per I,000 total births/live births at each gestation), Canada (excluding Ontario), 1991-97.

Birth weight-specific perinatal mortality rates (conventional calculation) declined sharply with increasing birth weight and showed the familiar crossover phenomenon; twins experienced a lower perinatal mortality rate compared with singletons at birth weights $<3,000 \mathrm{~g}$ and a higher perinatal mortality rate at 3,000 g and over (Figure 1). Similarly, gestational age-specific stillbirth rates, early neonatal death rates and perinatal mortality rates (conventional calculation) declined with increasing gestational age, and the mortality curves for singletons and twins intersected (Figure 2). For instance, at 35 weeks gestation the perinatal mortality rate among singletons $(24.7$ per 1,000 total births, Table 1) was more than two times as high as the rate among twins (11.1 per 1,000 total 
Table I: Numbers and rates of live births, stillbirths and early neonatal deaths among singletons births, Canada (excluding Ontario), 1991 to 1997.

\begin{tabular}{|c|c|c|c|c|c|c|c|c|c|c|}
\hline $\begin{array}{l}\text { Gestational } \\
\text { age }\end{array}$ & Stillbirths & Live births & $\begin{array}{c}\text { Early } \\
\text { neonatal } \\
\text { deaths }\end{array}$ & $\begin{array}{l}\text { Stillbirth } \\
\text { rate }(I) \dagger\end{array}$ & $\begin{array}{c}\text { Early neonatal } \\
\text { death } \\
\text { rate }(1) \dagger\end{array}$ & $\begin{array}{l}\text { Perinatal } \\
\text { mortality } \\
\text { rate }(1) \dagger\end{array}$ & $\begin{array}{c}\text { Fetuses at } \\
\text { risk }\end{array}$ & $\begin{array}{l}\text { Stillbirth } \\
\text { rate }(2) \dagger\end{array}$ & $\begin{array}{c}\text { Early neonatal } \\
\text { death } \\
\text { rate }(2) \dagger\end{array}$ & $\begin{array}{l}\text { Perinatal } \\
\text { mortality } \\
\text { rate }(2) \dagger\end{array}$ \\
\hline 32 & 288 & 4,287 & 125 & 63.0 & 29.2 & 90.3 & I,593,933 & 0.2 & 0.1 & 0.3 \\
\hline 33 & 314 & 5,758 & 118 & 51.7 & 20.5 & 71.1 & $1,589,358$ & 0.2 & 0.1 & 0.3 \\
\hline 34 & 329 & $10,66 \mid$ & 144 & 29.9 & 13.5 & 43.0 & I,583,286 & 0.2 & 0.1 & 0.3 \\
\hline 35 & 318 & 18,128 & 138 & 17.2 & 7.6 & 24.7 & I,572,296 & 0.2 & 0.1 & 0.3 \\
\hline 36 & 439 & 41,962 & 194 & 10.4 & 4.6 & 14.9 & $1,553,850$ & 0.3 & 0.1 & 0.4 \\
\hline 37 & 451 & 87,566 & 186 & 5.1 & 2.1 & 7.2 & $1,511,449$ & 0.3 & 0.1 & 0.4 \\
\hline 38 & 552 & 232,039 & 252 & 2.4 & 1.1 & 3.5 & $1,423,432$ & 0.4 & 0.2 & 0.6 \\
\hline 39 & 522 & 356,922 & 235 & 1.5 & 0.7 & 2.1 & I, $190,84 \mid$ & 0.4 & 0.2 & 0.6 \\
\hline 40 & 601 & 536,302 & 331 & I.I & 0.6 & 1.7 & 833,397 & 0.7 & 0.4 & I.I \\
\hline 41 & 304 & 245,665 & 152 & 1.2 & 0.6 & 1.9 & 296,494 & 1.0 & 0.5 & 1.5 \\
\hline$\geq 42$ & 92 & 50,433 & 58 & 1.8 & 1.2 & 3.0 & 50,525 & 1.8 & 1.2 & 3.0 \\
\hline Total $\neq$ & 8,694 & $|, 6| 4,53 \mid$ & 4,589 & 5.4 & 2.8 & 8.2 & $1,623,225$ & 5.4 & 2.8 & 8.2 \\
\hline
\end{tabular}

† Total births at each gestational week served as the denominator for stillbirth and perinatal mortality rates (I), while live births at each gestational week constituted the denominator for early neonatal death rates (I). Stillbirth, early neonatal death and perinatal mortality rates (2) were calculated using fetuses-at-risk as the denominator (see text). All rates are expressed per I,000. $\ddagger$ All gestational ages, including those $<32$ weeks and those with missing gestational age.

Table 2: Numbers and rates of live births, stillbirths and early neonatal deaths among twin births, Canada (excluding Ontario), I99 I to 1997.

\begin{tabular}{|c|c|c|c|c|c|c|c|c|c|c|}
\hline $\begin{array}{l}\text { Gestational } \\
\text { age }\end{array}$ & Stillbirths & Live births & $\begin{array}{c}\text { Early } \\
\text { neonatal } \\
\text { deaths }\end{array}$ & $\begin{array}{l}\text { Stillbirth } \\
\text { rate }(I) \dagger\end{array}$ & $\begin{array}{c}\text { Early neonatal } \\
\text { death } \\
\text { rate }(1) \dagger\end{array}$ & $\begin{array}{l}\text { Perinatal } \\
\text { mortality } \\
\text { rate }(1) \dagger\end{array}$ & $\begin{array}{c}\text { Fetuses at } \\
\text { risk }\end{array}$ & $\begin{array}{l}\text { Stillbirth } \\
\text { rate }(2) \dagger\end{array}$ & $\begin{array}{c}\text { Early neonatal } \\
\text { death } \\
\text { rate }(2) \dagger\end{array}$ & $\begin{array}{l}\text { Perinatal } \\
\text { mortality } \\
\text { rate }(2) \dagger\end{array}$ \\
\hline 32 & 33 & 1,182 & 20 & 27.2 & 16.9 & 43.6 & 31,821 & 1.0 & 0.6 & 1.7 \\
\hline 33 & 29 & $\mathrm{I}, 407$ & 14 & 20.2 & 10.0 & 29.9 & 30,606 & 0.9 & 0.5 & 1.4 \\
\hline 34 & 42 & 2,513 & 16 & 16.4 & 6.4 & 22.7 & 29,170 & 1.4 & 0.6 & 2.0 \\
\hline 35 & 28 & 3,302 & 9 & 8.4 & 2.7 & 11.1 & 26,615 & 1.1 & 0.3 & 1.4 \\
\hline 36 & 28 & 5,372 & 15 & 5.2 & 2.8 & 8.0 & 23,285 & 1.2 & 0.6 & 1.9 \\
\hline 37 & 29 & 6,835 & 10 & 4.2 & 1.5 & 5.7 & 17,885 & 1.6 & 0.6 & 2.2 \\
\hline 38 & 30 & 6,720 & 8 & 4.4 & 1.2 & 5.6 & $|I, 02|$ & 2.7 & 0.7 & 3.5 \\
\hline 39 & 15 & 2,843 & 7 & 5.2 & 2.5 & 7.7 & 4,271 & 3.5 & 1.6 & 5.2 \\
\hline 40 & 11 & 1,246 & 2 & 8.8 & 1.6 & 10.3 & 1,413 & 7.8 & 1.4 & 9.2 \\
\hline 41 & 1 & 129 & 0 & 7.7 & 0.0 & 7.7 & 156 & 6.4 & 0.0 & 6.4 \\
\hline$\geq 42$ & 0 & 26 & 0 & 0.0 & 0.0 & 0.0 & 26 & 0.0 & 0.0 & 0.0 \\
\hline Total & 703 & 34,944 & 753 & 19.7 & 21.5 & 40.8 & 35,647 & 19.7 & 21.5 & 40.8 \\
\hline
\end{tabular}

† Total births at each gestational week served as the denominator for stillbirth and perinatal mortality rates (I), while live births at each gestational week constituted the denominator for early neonatal death rates (I). Stillbirth, early neonatal death and perinatal mortality rates (2) were calculated using fetuses at risk as the denominator (see text). All rates expressed per I,000. $\ddagger$ All gestational ages, including those $<32$ weeks and those with missing gestational age.

births, Table 2). At 39 weeks gestation this relationship was reversed and the perinatal mortality rate was severalfold higher among twins (7.7 per 1,000 total births) than among singletons (2.1 per 1,000 total births).

Stillbirth, early neonatal death and perinatal mortality rates calculated using the alternative denominator (i.e., fetuses at risk for stillbirth, early neonatal death or perinatal death) are shown in Figure 3. Mortality rates (per gestational week) were markedly higher at later gestational ages among both singleton and twin births, although mortality increases occurred at an earlier gestational age among twins. Table 1 shows that the perinatal mortality rate among singletons increased nearly three-fold from 

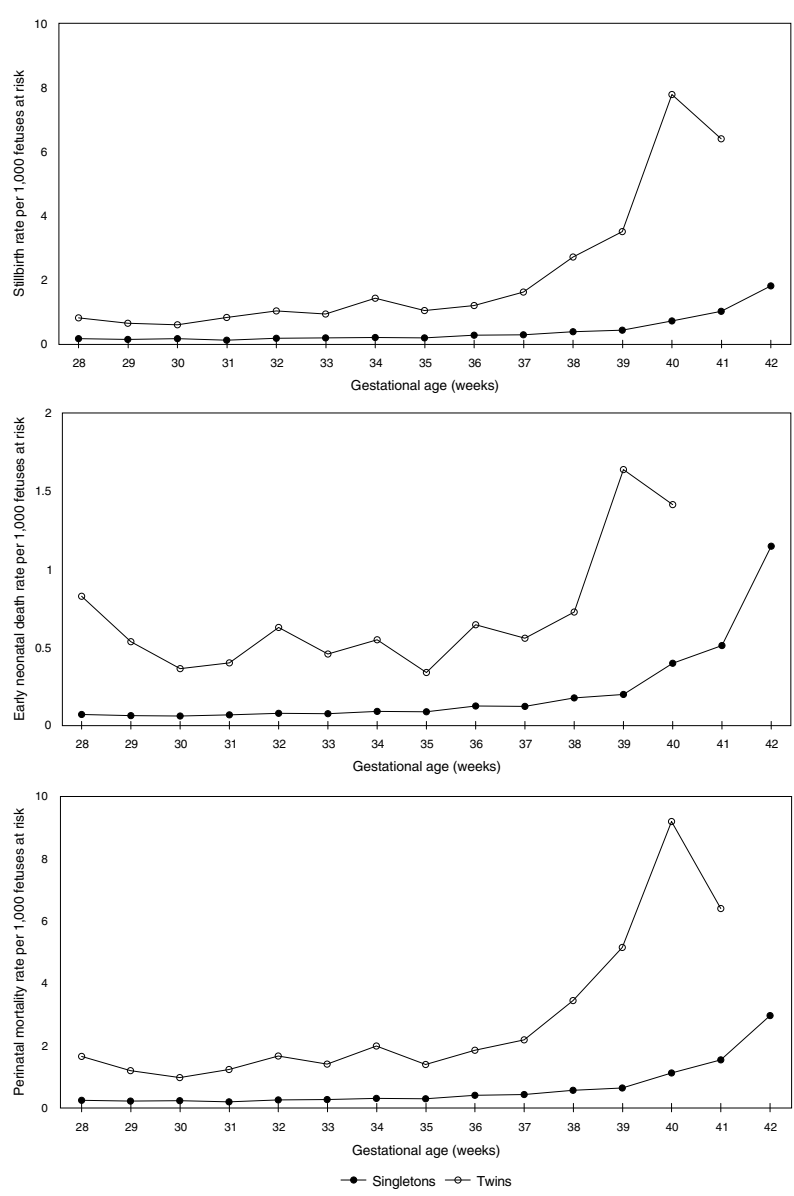

Figure 3

Stillbirth, early neonatal death and perinatal mortality rates among singleton and twin births (fetuses-at-risk approach). Legend text: Gestational age-specific stillbirth (upper panel), early neonatal death (middle panel) and perinatal mortality (lower panel) rates among singleton and twin births calculated using the fetuses-at-risk approach, Canada (excluding Ontario), 1991-97.

1.1 per 1,000 fetuses at risk at 40 weeks to 3.0 per 1,000 fetuses at risk at $\geq 42$ weeks gestation (relative risk at $\geq 42$ weeks vs 40 weeks $=2.7,95$ percent confidence interval [CI] 2.2 to 3.2). Similarly, among twins (Table 2), the perinatal mortality rate increased from 2.2 per 1,000 fetuses at risk at 37 weeks to 5.2 per 1,000 fetuses at risk at 39 weeks gestation (relative risk $2.4,95 \%$ CI 1.4 to 4.0 ). Stillbirth, early neonatal death and perinatal mortality curves did not intersect, however, when based on this alternative formulation of mortality risk. Birth weightspecific perinatal mortality curves, calculated using births at a given or higher birth weight category as the denomi- nator, also did not intersect (not shown). Similarly, gestational age- and birth weight-specific neonatal and infant mortality curves did not intersect when rates were calculated using the fetuses-at-risk approach.

Compared with singleton live births to nulliparous women, the gestational age distribution of singleton live births to parous women was slightly shifted to the left (except at preterm gestation, Figure 4). In contrast, the birth weight distribution of live births to nulliparous women was shifted to the left as compared with that of live births to parous women. Nulliparous women experienced a stillbirth rate of 6.2 per 1,000 total births, an early neonatal death rate of 2.9 per 1,000 live births and a perinatal mortality rate of 9.1 per 1,000 total births, while these mortality rates were 4.5 per 1,000 total births, 2.8 per 1,000 live births and 7.3 per 1,000 total births, respectively, among parous women. Between 32 and 35 weeks gestation, perinatal mortality rates (conventional calculation) among nulliparous women were lower than among parous women (Figure 4), while the reverse was true at gestational ages between 38 and 42 weeks. Birth weightspecific perinatal mortality curves also showed the expected crossover. When the number of fetuses at risk of perinatal death was used as the denominator for calculating gestational age-specific perinatal mortality rates, however, the curves did not intersect and nulliparous women had higher rates of perinatal mortality, especially at later gestational ages (Figure 4).

Gestational age-specific 'birth rates' presaged rising rates of perinatal death in the singletons-twins contrast. The same phenomenon was not observed when births to nulliparous women were compared with births to parous women (especially at term gestation, Figure 5). On the other hand, rising gestational age-specific rates of fetal growth-restriction preceded increases in gestational agespecific perinatal mortality rates in both the singletontwin and the nulliparous-parous contrasts.

\section{Discussion}

We have shown that perinatal mortality curves intersect if birth weight- and gestational age-specific perinatal mortality rates are based on the number of stillbirths and live births occurring at any particular birth weight or gestational age. Further, we have shown that this crossover in perinatal mortality rates is an artifact caused by an insufficient appreciation of the candidates at risk for perinatal death. Gestational age-specific perinatal mortality curves do not intersect if calculations are based on appropriate denominators, namely, on all fetuses at risk for perinatal death. This approach represents the appropriate epidemiologic formulation of risk since all fetuses at any gestation are at risk of stillbirth and/or neonatal death at that gestation [Joseph KS. Unpublished manuscript]. 

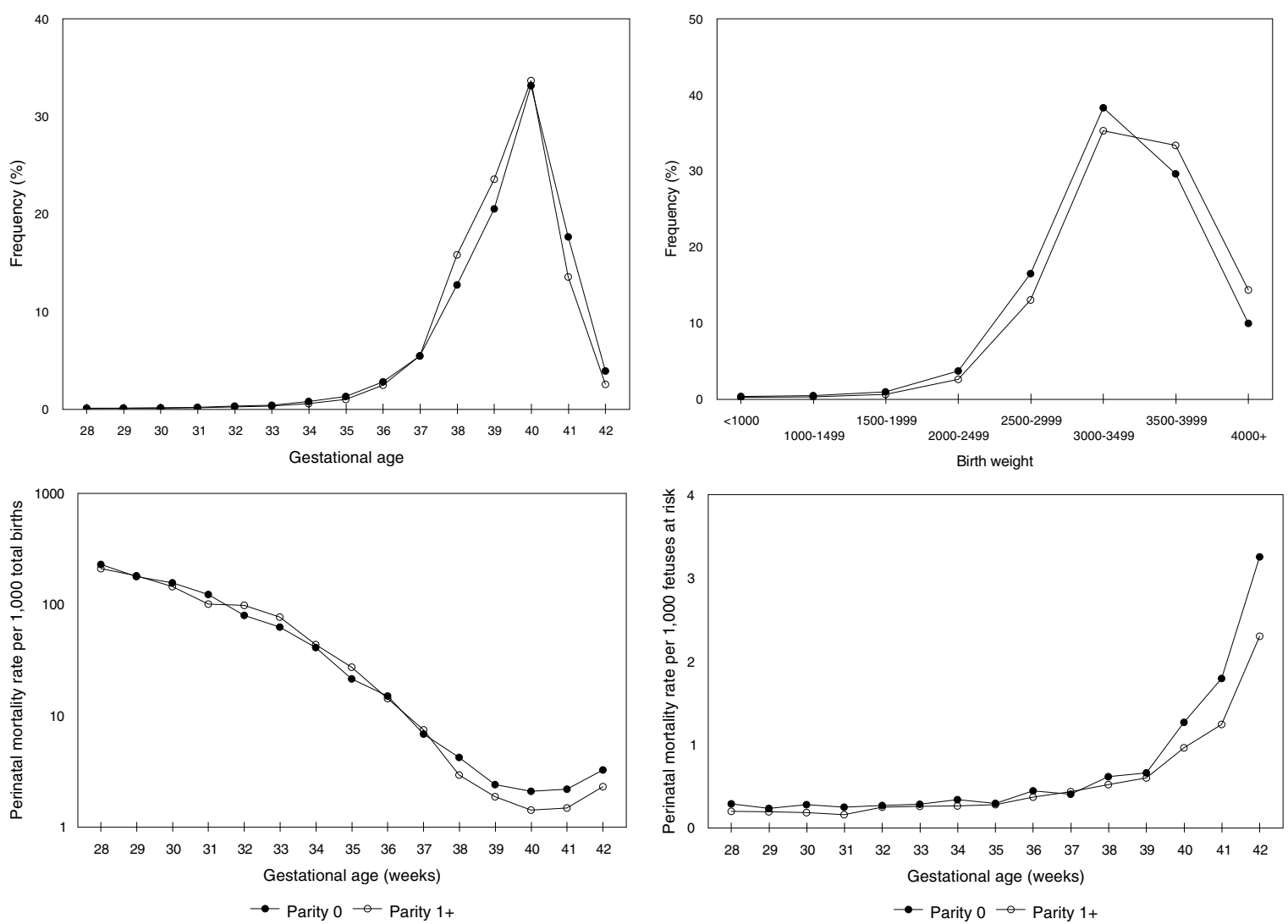

Figure 4

Gestational age and birth weight distributions and perinatal mortality rates among nulliparous and parous women. Legend text: Gestational age (upper left) and birth weight (upper right) distributions and gestational age-specific perinatal mortality rates, calculated as per conventional (lower left) and using the fetuses-at-risk approach (lower right), among births to nulliparous vs parous women, Canada (excluding Ontario), 199|-97.

Perinatal mortality curves (as calculated conventionally) intersect because the decline in the ratio of deaths to births is reversed at different gestational ages in dissimilar populations. This is a consequence of differences in the gestational age at which 'birth rates' and (true) perinatal mortality rates increases sharply. Relative birth weight and relative gestational age resolve the mortality crossover paradox because populations with left-shifted inflection points (at which birth and mortality rates rise) also have left-shifted birth weight and/or gestational age distributions.

Births to nulliparous women were shifted to the left with regard to birth weight but not gestational age (except at preterm gestation, Figure 4). In fact, fetal growth differ- ences between live births to nulliparous women and parous women are well recognized [21]. Despite this departure from the singletons-twins contrast, our analyses showed that use of appropriate denominators abolished the crossover in perinatal mortality rates. Figure 5 shows that increasing fetal growth-restriction rates presaged the rise in mortality in both the singletons-twins and nulliparous-parous comparisons. Fetuses in an relatively unfavorable uterine environment typically exhibit higher rates of growth-restriction and mortality; a left shift in the gestational age at which growth-restriction rates increase is attended by a left shift in the gestational age at which mortality rates increase (Figure 5). Analyses of birth data from the United States (1997 birth cohort) showed similar results in contrasts involving whites vs blacks and 

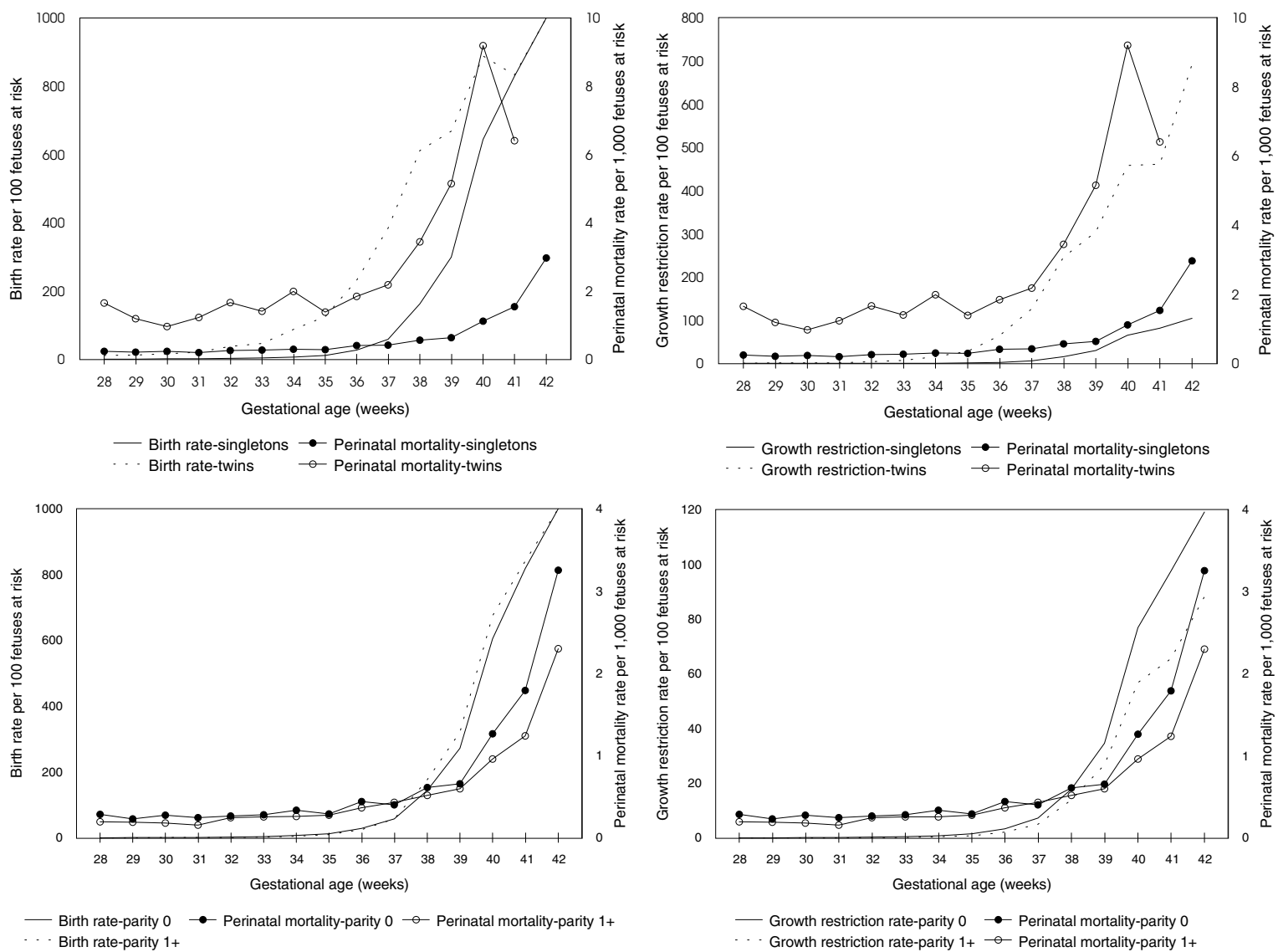

Figure 5

Birth, fetal growth-restriction and perinatal mortality rates among singletons vs twins and nulliparous vs parous women. Legend text: Gestational age-specific birth rates (left) and fetal growth-restriction rates (right) in relation to perinatal mortality rates among singletons vs twin births (upper) and among births to nulliparous vs parous women (lower), Canada (excluding Ontario) 1991-97.

nonsmokers vs smokers [Joseph et al. Unpublished manuscript]

Our conceptualization of the appropriate denominators for perinatal death is not new; the correct method of calculating stillbirth rates (risk) at different gestational ages was proposed over 15 years ago $[22,23]$, while the use of ongoing pregnancies as the denominator for neonatal and infant mortality was proposed about 5 years ago [24]. In recent years, we have carried out several studies [25-31] using appropriate denominators for gestational age-specific analyses in which the expected crossover in mortality was not evident despite demonstrated differences in gestational age and birth weight distributions. For instance, we showed no crossover in the gestational age- specific stillbirth rates among temporally distinct twin populations [27], no intersection in the gestational agespecific stillbirth curves of twins and triplets [29], and no late-gestation crossover in feto-infant mortality rates due to congenital anomalies among Canadian births in recent vs earlier periods [26]. Similarly, others have demonstrated no cross over in stillbirth rates (using fetuses at risk as the denominator) among whites and blacks [23].

The risk of perinatal death increases with increasing gestational age. It is not difficult for obstetricians to reconcile the increases in stillbirth rates with advancing gestation, since selective early delivery to prevent stillbirth (in highrisk pregnancies) has been a cornerstone of modern obstetrics [30,32]. Accepting that early neonatal death 
rates increase as gestational age advances may present a challenge, especially for neonatologists, given their experience with very early gestation vs term live births. Adopting the proper clinical/biologic viewpoint in this context involves a change in perspective from that of live-born infants to that of ongoing pregnancies or fetuses at risk of perinatal death (the latter term avoids a minor denominator problem introduced by multiple pregnancies).

Several clinical and epidemiologic implications and caveats emerge from our findings. First, our finding of increasing perinatal mortality with increasing gestational ages provides the basis for developing a theoretical justification for recent increases in obstetric intervention at the population level. Increases in labor induction and cesarean delivery rates at preterm, term and post-term gestation $[30,33,34]$ are supported by empirical evidence but not by contemporary obstetric theory (under which perinatal mortality rates decrease with increasing gestational age). Our alternative formulation does not justify indiscriminate obstetric intervention at any gestation however, although it does support selective early delivery in highrisk pregnancy. Our findings also raise concerns about the assumptions used in the creation of fetal growth standards. The use of separate fetal growth standards by plurality [35], parity [21], and other factors may missclassify growth-restricted fetuses at risk for perinatal death.

With regard to epidemiologic and statistical modeling in perinatal research, the appropriate conceptualization of the risk set mandates a reexamination of the role of gestational age (and birth weight). Logistic regression modeling of perinatal mortality with gestational age (or birth weight) as a determinant implies the conventional denominators (total births at that gestational age or birth weight). Mortality crossover is implicit in such a model; the effect of plurality, maternal smoking, etc will represent an average of the opposing effects observed across gestational age categories (unless otherwise specified). Although gestational age at delivery is highly predictive of death, it should only be used as a determinant in causal regression modeling on an ad hoc basis, since gestational age lies in the pathway between fetal compromise and death. It should also be noted that measures such as gestational age-specific perinatal mortality, extended perinatal mortality (stillbirths plus neonatal deaths) and fetoinfant mortality are based on two time-scale anchors (last menstrual period and birth) and are preferably replaced by indices constructed using a time scale with a single anchor (such as post-menstrual or post-conceptional age [36]). Finally, population differences in the gestational age at which perinatal mortality rates increase sharply (eg., among singletons vs twins) imply that gestational age-specific incidence (hazard) rates of perinatal death may not be proportional.
Our study has the limitations that are typical of studies involving information from large databases. The gestational age of stillbirths typically represents the physiciandetermined gestational age at delivery rather than gestational age at death. The difference (although systematically overestimated) is unlikely to be large [22], especially in recent years, except for twin pregnancies in which one fetus dies early. This is not a serious limitation in the context of our study, however, as the crossover in perinatal mortality rates occurred despite this problem (Figure 2). The approximate nature of our estimates of gestational age-specific rates of growth- restriction (which are based on small-for-gestational age live births and represent rates of 'revealed' growth-restriction) is another study weakness. Other limitations include potential errors in gestational age, inconsistencies due to data transcription, and missing information on a small fraction of births. Gestational age was missing among 12,205 of $1,649,476$ $(0.7 \%)$ live births and among 210 of 9,397 (2.2\%) stillbirths. Birth weight was missing among 6,599 (0.4\%) live births and 695 (7.4\%) stillbirths.

\section{Conclusions}

We have provided a parsimonious explanation for intersecting perinatal mortality curves which involves a reexamination of the epidemiologic issues pertinent to determining the rate of perinatal death at any given gestation. While our conceptualization of the candidates at risk of perinatal death merely invokes or extends previous epidemiologic formulations for mortality risk [22-24], it has important clinical and epidemiologic implications for conceptualizing perinatal risks more generally.

\section{Competing interests}

None

\section{Authors' contributions}

KSJ proposed the solution and drafted the manuscript. SL and KSJ carried out the analysis. KD independently deduced the solution for the stillbirth crossover phenomenon. All authors contributed to revising the manuscript for intellectual content. The draft manuscript was then discussed at a meeting of the Fetal and Infant Health Study Group of the Canadian Perinatal Surveillance System and further revised. All authors read and approved the final version.

\section{Acknowledgments}

We are grateful to the Vital Statistics Registrars of the provinces and territories for providing us with access to the data. Dr. Joseph is supported by a Clinical Research Scholarship from the Dalhousie University Faculty of Medicine and the Peter Lougheed/CIHR New Investigator award from the Canadian Institutes of Health Research. Dr. Ananth is partially supported by a grant (ROI) from the National Institute of Child Health and Human Development, National Institutes of Health. Dr. Wen, Dr. Platt and Dr. Kramer are career scientists of the Canadian Institutes of Health Research. 


\section{References}

I. Yerushalmy J: The relationship of parents' cigarette smoking to outcome of pregnancy - implications as to the problem of inferring causation from observed associations Am J Epidemio I97|, 93:443-456.

2. Meyer MB and Comstock GW: Maternal cigarette smoking and perinatal mortality Am J Epidemiol 1972, 96: I- I0.

3. Lie RT: Invited commentary: Intersecting perinatal mortality curves by gestational age - are appearances deceiving? $\mathrm{Am}$ Epidemiol 2000, I52: I I 17-III9.

4. Wilcox AJ and Russell IT: Birthweight and perinatal mortality: III. Towards a new method of analysis Int J Epidemiol 1986 I5: 188-196.

5. English PB and Eskenazi B: Reinterpreting the effects of maternal smoking on infant birthweight and perinatal mortality: a multivariate approach to birth weight standardization Int Epidemiol 1992, 21:1097-I I05.

6. Wilcox AJ and Skjœrven R: Birth weight and perinatal mortality: the effect of gestational age Am J Public Health 1992, 82:378382.

7. Wilcox AJ: Birth weight and perinatal mortality: the effect of maternal smoking Am I Epidemiol 1993, I37: I098-I I04.

8. Buekens $P$ and Wilcox AJ: Why do small twins have a lower mortality than small singletons? Am J Obstet Gynecol 1993 |68:937-94|.

9. Wilcox AJ, Skjœrven R, Buekens P and Kiely J: Birth weight and perinatal mortality: A comparison of the United States and Norway JAMA 1995, 272:709-7I I

10. Hertz-Picciotto $I$ and Din-Dzietham R: Comparisons of infant mortality using a percentile-based method of standardization for birthweight or gestational age Epidemiol 1998, 9:6 I-67.

II. Cheung YB, Yip P and Karlberg J: Mortality of twins and singletons by gestational age: a varying-coefficient approach $\mathrm{Am} \mathrm{J}$ Epidemiol 2000, I52: I 107-I I16.

12. Mittendorf R, Williams MA, Kennedy JL Jr, Berry RE, Herschel M, Aronson MP and Davidson KM: A hypothesis to explain paradoxical racial differences in neonatal mortality Am J Prev Med 1993 9:327-330.

13. Platt RW, Yang H, Kramer MS and Joseph KS: Maturity, growth and infant mortality: a new approach [abstract] Paediatric Perinat Epidemiol 2000, I4:A25.

14. Fair ME and Cyr M: The Canadian birth database: a new research tool to study reproductive outcomes Health Rep 1993, 5:281-290.

I5. Joseph KS, Kramer MS, Marcoux S, Ohlsson A, Wen SW, Allen AC and Platt $R$ : Determinants of preterm birth rates in Canada from 198I through 1983 and from 1992 through $1994 \mathrm{New}$ Engl J Med 1998, 339:1434-9.

16. Kramer MS, Demissie K, Yang H, Platt RW, Sauve R, Liston R and for the Fetal and Infant Health Study Group of the Canadian Perinatal Sur veillance System: The contribution of mild and moderate preterm birth to infant mortality JAMA 2000, 284:843-9.

17. Fair M, Cyr M, Allen AC, Wen SW, Guyon G and Macdonald RC: Validation study for a record linkage of births and infant deaths in Canada Ottawa, Canada: Statistics Canada. Catlogue No. 84F00 I3XIE I 999.

18. Joseph KS and Kramer MS: Recent trends in infant mortality rates and proportions of low-birth-weight live births in Canada CMAJ 1997, I 57:535-54I.

19. Rothman KJ and Greenland S: Modern epidemiology LippincottRaven Publishers. Philadelphia 1998:287-89.

20. Kramer MS, Platt RW, Wen SW, Joseph KS, Allen A, Abrahamowicz $M$, Blondel B and Breart G: A new and improved populationbased reference for birth weight for gestational age Pediatrics 2001, 1 08:e35.

21. Zhang J and Bowes WA Jr: Birth-weight-for gestational age patterns by race, sex, and parity in the United States Obstet Gynecol 1995, 86:200-208.

22. Yudkin PL, Wood $L$ and Redman CWG: Risk of unexplained stillbirth at different gestational ages Lancet 1987, I: | | $92-$ I I94.

23. Ferguson $\mathrm{R}$ and Myers SA: Population study of the risk of fetal death and its relationship to birth weight, gestational age, and race Am J Perinatol 1994, I I:267-272.

24. Hilder L, Costeloe $\mathrm{K}$ and Thilaganathan B: Prolonged pregnancy: evaluating gestation-specific risks of fetal and infant mortality Br j Obstet Gynecol 1998, 105:169-173.
25. Joseph KS, Allen AC, Dodds L, Vincer MJ and Armson BA: Causes and consequences of recent increases in preterm birth among twins Obstetrics Gynecol 200I, 98:57-64.

26. Liu S, Joseph KS, Wen SW, Kramer MS, Marcoux S, Ohlsson A and Sauve R: Changing patterns of fetal and infant death due to congenital anomalies in Canada Am J Med Genetics 200I, I 04:713.

27. Joseph KS, Marcoux S, Ohlsson A, Liu S, Allen AC, Kramer MS and Wen SW: Changes in stillbirth and infant mortality associated with increases in preterm birth among twins Pediatrics 200I, I08: |055-106|

28. Liu S, Joseph KS, Kramer MS, Allen AC, Sauve R, Rusen ID and Wen SW: The impact of prenatal diagnosis and pregnancy termination on overall infant mortality rates JAMA 2002, 287: I56I1567.

29. Joseph KS, Marcoux S, Ohlsson A, Kramer MS, Allen AC, Liu S, Wen SW, Demissie K, Sauve R and Liston R: Preterm birth, stillbirth and infant mortality among triplet births in Canada, I 985-96 Paediatr Perinatal Epidemiol 2002, I 6: | 4 | - | 48

30. Joseph KS, Demissie K and Kramer MS: Trends in obstetric intervention, stillbirth and preterm birth Semin Perinatol 2002, 26:250-259

31. Liu S, Joseph KS and Wen SW: Trends in fetal and infant deaths due to congenital anomalies Semin Perinatol 2002, 26:268-276.

32. O'Dowd MJ and Philipp EE: The history of obstetrics and gynecology Parthenon Publishing Group. New York 1994.

33. Yawn BP, Wollan P, McKeon $\mathrm{K}$ and Field CS: Temporal changes in rates and reasons for medical induction of term labor, 1980 . I996 Am J Obstet Gynecol 200 I, I 84:6 I I-6I 9.

34. Ventura SJ, Martin JA, Curtin SC, Menacker F and Hamilton BE: Births: Final data for 1999 National vital statistics reports. Hyattsville, Maryland: National Centre for Health Statistics. 200I, 49(I):

35. Alexander G, Himes J, Kaufman R, Mor J and Kogan M: A United States national reference for fetal growth Obstet Gynecol 1996 , 87:163-168.

36. Scott DT and Tyson JE: Follow up of infants discharged from newborn intensive care In: Oski's pediatrics - principles and practice Edited by: McMillan JA, DeAngelis CD, Feigin RD, Warshaw JB. Lippincott Williams \& Wilkins. New York; 1999:209-214.

\section{Pre-publication history}

The pre-publication history for this paper can be accessed here:

http://www.biomedcentral.com/1471-2393/3/3/prepub

Publish with BioMed Central and every scientist can read your work free of charge

"BioMed Central will be the most significant development for disseminating the results of biomedical research in our lifetime. "

Sir Paul Nurse, Cancer Research UK

Your research papers will be:

- available free of charge to the entire biomedical community

- peer reviewed and published immediately upon acceptance

- cited in PubMed and archived on PubMed Central

- yours - you keep the copyright
BioMedcentral 